\title{
DNS and LES of primary atomization of turbulent liquid jet injection into a gaseous crossflow environment
}

\author{
Anirudh Asuri Mukundan ${ }^{\mathrm{a}}$, Giovanni Tretola ${ }^{\mathrm{b}, \mathrm{c}}$, Thibaut Ménard ${ }^{\mathrm{a}, \mathrm{d}, *}$, \\ Marcus Herrmann ${ }^{\mathrm{e}}$, Salvador Navarro-Martinez ${ }^{\mathrm{b}, *}$, Konstantina Vogiatzaki ${ }^{\mathrm{c}}$, \\ Jorge César Brändle de Motta ${ }^{\mathrm{a}, \mathrm{d}}$, Alain Berlemont ${ }^{\mathrm{a}}$ \\ ${ }^{a}$ CNRS UMR6614-CORIA, Saint Etienne du Rouvray, 76801, France \\ ${ }^{b}$ Imperial College London, 58 Princes Gate, Kensington, London SW7 $1 A L, U K$ \\ ${ }^{c}$ Advanced Engineering Centre, University of Brighton, Brighton BN2 4GJ, UK \\ ${ }^{d}$ Université de Rouen Normandie, Saint Etienne du Rouvray, 76801, France \\ ${ }^{e}$ School for Engineering of Matter, Transport and Energy, Arizona State University, \\ P.O. Box 876106, Tempe, AZ 85287-6106, USA
}

\begin{abstract}
In this paper, we study the primary atomization characteristics of liquid jet injected into a gaseous crossflow through direct numerical simulations (DNS) and large eddy simulations (LES). The DNS use a coupled level set volume of fluid (CLSVOF) sharp interface capturing method resolving all relevant scales to predict the drop size distribution (DSD) for drops larger than the grid spacing. The LES use a volume of fluid (VOF) diffused interface method modelling the sub grid droplets. The purpose of this paper is to provide a comparison of the results of drop data between DNS and LES. The simulations are performed for a liquid jet injection with liquid-gas momentum flux ratio of 6.6 , liquid jet Reynolds number of 14,000 injected
\end{abstract}

\footnotetext{
*Corresponding author:

Email addresses: thibaut.menard@coria.fr (Thibaut Ménard), s.navarro@imperial.ac.uk (Salvador Navarro-Martinez)
} 
into a crossflowing air with Reynolds number 570,000 and Weber number of 330 at a liquid-to-gas density ratio of 10 . Two distinct and simultaneous atomization/breakup mechanisms have been observed in the simulations: column/bag breakup and ligament/surface breakup. It was found that the DSDs obtained from the DNS and LES each follow a log-normal distribution based on their respective droplet diameter data. An overlap region exists between the individual DSDs from the DNS and LES when combined. The width of this overlap region decreases along the downstream direction. A log-normal distribution is found to be a good fit to the combined DSD incorporating both resolved and sub-grid droplets. This information is relevant for the secondary atomization simulations and modeling.

Keywords:

Atomization, Crossflow, Interface capture, Stochastic fields, Drop size distribution

\section{Introduction}

With the growing number of long-haul passenger aircraft, it has become necessary to develop more efficient aircraft engines that can offer long range with reduced pollutant emissions. The amount of pollutants produced depends on the completeness of the fuel combustion which is linked to the quality of injected liquid fuel atomization. Atomization is the process of disintegration of the coherent liquid structure into droplets. In fact, small droplets promote quick evaporation and better mixing with the oxidizer, while large drops may deposit on the walls of the engine chamber thereby lowering the combustion efficiency and eventually harming the engine. Hence, 
it is imperative to understand and gain control of the atomization process.

To that end, a commonly employed injection configuration in aero engines called liquid jet in crossflow is chosen for the investigation in this study. In this configuration, the liquid fuel injected into a crossflowing gaseous environment atomizes into droplets. There have been multiple experimental investigations [1, 2] for this configuration to understand the breakup mechanisms [3], to classify the breakup regimes [4], to characterize the jet penetration [5, 6], and to gain understanding of the jet dynamics [7]. For a complete review, the reader is referred to the work of Broumand and Birouk [8].

There have also been several numerical studies, for example, Herrmann [9] performed detailed numerical simulations of primary atomization of an experimentally investigated liquid jet in crossflow configuration [3]. The grid dependence of the droplet diameters [9], the effect of the density ratio on the atomization characteristics [10] and the jet dynamics [11] have been investigated in the past. The effect of inflow velocity profiles on the atomization characteristics have been investigated by Ghods and Herrmann [12] using three different velocity profiles (in-nozzle large eddy simulations (LES), fully developed turbulent velocity profiles each with, and without nozzle geometry) under two different density ratio operating conditions. These studies found that the jet penetration was in agreement with the experimental correlation of $\mathrm{Wu}$ et al. [5] for the case of turbulent inflow velocities taking the injector geometry into account. Furthermore, they found that the liquid column was more deformed when using a fully developed turbulent pipe inflow without nozzle geometry than when including the nozzle geometry. The results confirm the observations of Brown and McDonell [3] that the 
initial velocity profile has a high impact on the atomization characteristics. Owkes et al. [13] used LES to investigate the effect of rounded and sharp edged nozzle exits on the atomization characteristics. They found excellent agreement between the simulations and experiments of Gopala [14] for both the nozzle geometries and reasonable match of the droplet sizes with experimental data. Recently, Li and Soteriou [15, 16] investigated the effects of liquid fuel density, viscosity, and intermeditate Weber number on the liquid jet penetration, evolution of Sauter Mean Diameter (SMD) as a function of crossflow direction, and jet dynamics of the crossflow atomization.

The direct numerical simulations (DNS) approach provides a complete description of the flow dynamics, but its high computational cost limits its use to flows with moderate Reynolds and Weber numbers and liquid-gas density ratio. LES on the other hand has cheaper computational cost but requires modeling of the sub grid (or unresolved) multiphase turbulence dynamics. Despite the numerous efforts, there has not been a direct comparison of DNS and LES for the liquid jet in crossflow injection configuration. This paper presents, for the first time, the direct comparison of results of primary atomization of turbulent liquid jet injected into a subsonic gaseous crossflow. The DNS in this study is to be considered as quasi-DNS without sub-grid scale model in capturing the small droplets (due to under-resolution). However, it resolves all relevant processes [17, 18] to predict drop sizes larger than the mesh spacing but does not resolve the Kolmogorov scales for both the phases in a classical sense of DNS. The operating condition chosen in this work is comparable to that of a practical aero engine. The non-dimensional numbers such as liquid-gas momentum flux ratio, Reynolds, and Weber numbers 
follow the experimental study of Brown and McDonell [3]. This study aims to investigate and contrast the different atomization and breakup characteristics, jet penetration, and drop size distributions (DSDs) between DNS and LES.

This paper is organized as follows. The governing equations solved in DNS and LES along with the numerical methods of the respective flow solvers and phase interface capturing methods are presented in Section 2. This is followed by the presentation of the liquid jet in crossflow configuration, case setup, and operating conditions in Section 3. Finally, the results obtained from the simulations are presented and discussed in Section 4 from which the conclusions are drawn in Section 5.

\section{Governing Equations and Numerical Methods}

\section{1. $D N S$}

To describe the multiphase flow with DNS, the pressure and velocity fields of the flow are obtained by solving the following conservative form of the incompressible Navier-Stokes equations,

$$
\begin{aligned}
& \nabla \cdot \boldsymbol{u}=0 \\
& \frac{\partial(\rho \boldsymbol{u})}{\partial t}+\nabla \cdot(\rho \boldsymbol{u} \boldsymbol{u})=-\nabla P+\nabla \cdot(2 \mu \boldsymbol{D})+\boldsymbol{T}_{\sigma}
\end{aligned}
$$

where $\rho$ is the density, $\boldsymbol{u}$ is the velocity field, $P$ is the pressure field, $\mu$ is the

dynamic viscosity, $\boldsymbol{D}=\frac{1}{2}\left(\boldsymbol{\nabla} \boldsymbol{u}+\boldsymbol{\nabla}^{T} \boldsymbol{u}\right)$ is the strain rate tensor and $\boldsymbol{T}_{\sigma}$ is the surface tension force acting on the location of the liquid/gas interface $\boldsymbol{x}_{\text {intf }}$.

The interface is captured using a coupled level set volume of fluid (CLSVOF) method [19] in which the location of the interface $\boldsymbol{x}_{\text {intf }}$ is described by a scalar 
level set signed distance function $\phi$ proposed by Osher and Sethian [20]. The value of $\phi(\boldsymbol{x}, t)>0$ defines the liquid phase (fluid 1 ), $\phi(\boldsymbol{x}, t)<0$ defines the gas phase (fluid 2), and $\phi\left(\boldsymbol{x}_{\mathrm{intf}}, t\right)=0$ defines the location of the interface. The fraction of the liquid volume within a computational cell is represented by the liquid volume fraction scalar $F$. The advection of the interface is achieved by solving the following transport equation for $\Psi=[\phi, F]^{T}$ :

$$
\frac{\partial \Psi}{\partial t}+\boldsymbol{u} \cdot \nabla \Psi=0
$$

The physical properties of the phases $\alpha$ in a compuational cell is determined using $F$ as $\alpha(\boldsymbol{x})=\alpha_{1} F(\boldsymbol{x})+\alpha_{2}(1-F(\boldsymbol{x}))$. This expression involves an assumption that the physical properties are constant within each phase where $\alpha$ is either density $\rho$ or viscosity $\mu$ with the indices 1 and 2 denoting the respective fluid properties. A directionally-split advection method [21] is used to solve Equation (3). This approach is implemented in the in-house flow solver ARCHER [19, 22, 23] with consistent mass and momentum flux computation [22. A staggered variable arrangement with scalar quantities stored at the cell center and vector quantities at the cell faces is employed in ARCHER. A second-order central difference scheme is employed for discretization of the spatial derivatives and fifth-order WENO scheme for convective term with second-order Runge-Kutta scheme for temporal advancement of the NavierStokes equations. For more details, the reader is referred to Vaudor et al. [22. The physics of atomization of the coherent liquid jet into droplets of varying sizes are captured by solving these equations. All the droplets are described in the Eulerian framework and no transfer to Lagrangian descriptions is employed in the DNS. 


\section{2. $L E S$}

In the case of LES, a $\Sigma$ - $Y$-PDF approach is employed to solve the incompressible Navier-Stokes equations. This approach consists of solving the joint probability density function (jPDF) transport equation of liquid volume and surface density using stochastic methods [24]. A system of stochastic partial differential equations (SPDE) is derived from the PDF transport equations using $N$ stochastic fields, where each stochastic field has its own liquid volume fraction $F^{n}$ (instead of the mass fraction $Y^{n}$ ) and surface density $\Sigma^{n}$ where $n \in\{1,2, \ldots N\}$. The advantage of this approach is that it can simulate both dense and dilute regions of the spray.

Using the Ito formulation [25], the following transport equations for the stochastic fields are obtained,

$$
\begin{aligned}
& \frac{d F^{n}}{d t}+\bar{u}_{j} \frac{\partial F^{n}}{\partial x_{j}}=\frac{\partial}{\partial x_{j}}\left[D_{\mathrm{SGS}} \frac{\partial F^{n}}{\partial x_{j}}\right]+\sqrt{2 D_{\mathrm{SGS}}} \frac{\partial F^{n}}{\partial x_{j}} \frac{d W_{j}^{n}}{d t}, \\
& \frac{d \Sigma^{n}}{d t}+\bar{u}_{j} \frac{\partial \Sigma^{n}}{\partial x_{j}}=\frac{\partial}{\partial x_{j}}\left[D_{\mathrm{SGS}} \frac{\partial \Sigma^{n}}{\partial x_{j}}\right]+\sqrt{2 D_{\mathrm{SGS}}} \frac{\partial \Sigma^{n}}{\partial x_{j}} \frac{d W_{j}^{n}}{d t}+S
\end{aligned}
$$

where $S$ is the source term, $D_{\mathrm{SGS}}$ is the sub-grid scale (SGS) diffusivity which is proportional to the SGS viscosity $\nu_{\mathrm{SGS}}$ according to the relation $D_{\mathrm{SGS}}=\nu_{\mathrm{SGS}} / \mathrm{Sc}_{\mathrm{SGS}}$ with a SGS Schmidt number of $\mathrm{Sc}_{\mathrm{SGS}}=1$. In this equation, $d \mathbf{W}^{n}$ represents a Wiener process with mean 0 and variance equal to $\Delta t^{1 / 2}$. The solution of Equations (4) and (5) allows to obtain the moments of the PDF equations. The first-moments are computed as

$$
\bar{F}=\frac{1}{N} \sum_{n=1}^{N} F^{n}, \quad \bar{\Sigma}=\frac{1}{N} \sum_{n=1}^{N} \Sigma^{n} .
$$

If all the moments are known, Equations (4) and (5) are equivalent to the LES filtered counterpart [24]. After the stochastic field equations have been 
advanced in time, all relevant parameters can be obtained directly. For example, the characteristic fragment length per stochastic field is the Sauter Mean Diameter (SMD) is defined as

$$
d_{32}^{n}=6 \frac{F^{n}\left(1-F^{n}\right)}{\Sigma^{n}}
$$

where the corresponding filtered moment $\bar{d}_{32}$ can be obtained directly from Equation (6). The DSD (in space and time) can then be obtained directly from binning the SMD values.

The source term $S$ is modelled as a non-linear restoration to equilibrium term as proposed by Lebas et al. [26],

$$
S=\frac{\Sigma^{n}}{\tau}\left(1-\frac{\Sigma^{n}}{\Sigma_{\mathrm{eq}}}\right),
$$

where $\Sigma_{\text {eq }}$ is the equilibrium surface density and $\tau$ is the associated relaxation time-scale related to the flow [24]. At high Weber numbers typical of spray atomization, the time scale can be LES-scale based on the filtered strain rate, $\tau \propto\left\|\bar{S}_{i j}\right\|^{-1}$, or proportional to a turbulent time scale (as in the Reynolds Averaged Navier-Stokes approach). Capillary effects in the source term are restricted to the local value of $\Sigma_{\text {eq }}$, which can be characterized by a critical Weber number [27] as $\mathrm{We}_{\text {crit }}=2 k_{\mathrm{SGS}}\left(\rho_{l}+\rho_{g}\right) F(1-F) /\left(\sigma \Sigma_{\text {eq }}\right)$, where $k_{\mathrm{SGS}}$ is the local sub-grid scale turbulent kinetic energy and following NavarroMartinez [24], $\mathrm{We}_{\text {crit }}=1$ is used in this work.

The $\Sigma$ - $Y$-PDF approach is implemented in the finite volume open source software OpenFOAM [28]. The spatial derivatives for the momentum equation are approximated by standard second-order central differences. The momentum equations are integrated using a second-order Crank-Nicolson temporal scheme. The WALE [29] method is used to model the sub grid stress 
terms using $\nu_{\text {SGS }}$ since it did not introduce excessive diffusion for this configuration. The stochastic fields are solved using an operator-splitting technique with the MULES scheme [30] for the convective step to minimise numerical diffusion and central derivatives for diffusive processes. The Ito formulation is retained in this work. The spatial gradient appearing in the stochastic terms of Equations (4) and (5) in the Ito formulation is approximated using central differences. The temporal term of the Ito process is discretized using the Euler-Maruyama scheme [31]. The Wiener process is modelled with a weak approximation as $d W_{j}^{n}=\eta_{j}^{n} \Delta t^{1 / 2}$, where $\eta_{i}^{n}$ is a $\{-1,1\}$ dichotomic random vector [32]. The resultant scheme is weakly consistent of order $\Delta t^{1 / 2}$ [31] and the number of fields chosen in the simulations is $N=16$ following Navarro-Martinez [24]. Similar to DNS, no Lagrangian transformation of droplets is performed in the LES.

\section{Operating Condition and Computational Domain}

The operating condition for the simulations (both DNS and LES) used in this work has been studied experimentally by Brown and McDonell [3] and numerically by Herrmann [9] previously. Table 1 summarizes the values of the physical quantities of the liquid and gas phase. It is to be remarked that although the liquid-to-gas density ratio $r_{\rho}$ is artificially reduced in the simulations (by modifying liquid velocity, viscosity, and density), all the nondimensional numbers, i.e., liquid-gas momentum flux ratio $q$, Reynolds number Re, and Weber number We of the liquid and gas phases remain the same as in the experiments. It is to be noted that all the details mentioned in this section apply to both DNS and LES unless otherwise stated explicitly. 
Table 1: Operating conditions and non-dimensional numbers

\begin{tabular}{lc}
\hline \hline Quantity & Simulation \\
\hline Jet diameter $\left(D_{j}\right)[\mathrm{mm}]$ & 1.3 \\
Jet density $\left(\rho_{j}\right)\left[\mathrm{kg} / \mathrm{m}^{3}\right]$ & 12.25 \\
Jet velocity $\left(U_{j}\right)[\mathrm{m} / \mathrm{s}]$ & 97.84 \\
Jet viscosity $\left(\mu_{j}\right)[\mathrm{kg} / \mathrm{ms}]$ & $1.11 \times 10^{-4}$ \\
Surface tension $(\sigma)[\mathrm{N} / \mathrm{m}]$ & 0.07 \\
Crossflow gas density $\left(\rho_{c}\right)\left[\mathrm{kg} / \mathrm{m}^{3}\right]$ & 1.225 \\
Crossflow gas velocity $\left(u_{c}\right)[\mathrm{m} / \mathrm{s}]$ & 120.4 \\
Crossflow viscosity $\left(\mu_{c}\right)[\mathrm{kg} / \mathrm{ms}]$ & $1.82 \times 10^{-5}$ \\
& \\
Density ratio $\left(r_{\rho}\right)$ & 10 \\
Momentum flux ratio $(q)$ & 6.6 \\
Jet Weber number $\left(\mathrm{We}_{j}\right)$ & 2178 \\
Jet Reynolds number $\left(\mathrm{Re}_{j}\right)$ & 14,079 \\
Crossflow Weber number $(\mathrm{We})$ & 330 \\
Crossflow Reynolds number $\left(\mathrm{Re}_{c}\right)$ & 570,000 \\
\hline \hline
\end{tabular}

The computational domain chosen in this work is of size $40 D_{j} \times 10 D_{j} \times$ $20 D_{j}$ [17] where $D_{j}$ is the diameter of the liquid jet. This domain is smaller than in the experiments [3], however, as observed from previous numerical studies [9-11], the domain size reduction does not affect the primary breakup description.

In the case of DNS, a uniform structured Cartesian mesh containing about 262 million cells is used for discretizing the computational domain. This re- 
sults in a uniform mesh spacing of $\Delta x=\Delta y=\Delta z=D_{j} / 32$ throughout the domain. While in the case of LES, a Cartesian mesh composed of approximately 2.5 million cells is employed. The grid is refined in the region close the liquid inlet and then stretched (at 3\% cell expansion ratio) along the crossflow downstream direction resulting in a computational cell size $\Lambda$ in the range of $D_{j} / 32$ to $D_{j} / 8$ with $\Lambda_{\min }=D_{j} / 32$ near the injection region. Since an implicit filtering technique with the computational mesh as filter is employed in LES, the filter width is the cubic root of the computational cell volume.

Both the DNS and LES use a fully developed turbulent pipe flow velocity profile at the inlet of the liquid jet. The liquid jet at $t^{*}=t U_{j} / D_{j}=0$ is initialized in the DNS as a cylinder of diameter $D_{j}$ and height $4 \Delta x$ protruding into the crossflow channel. While in LES, the initial domain is empty at $t^{*}=0$ and the injection starts from $t^{*}>0$. The reported results from the simulations in the following sections are non-dimensionlized using the jet velocity $U_{j}$ and jet diameter $D_{j}$ as reference quantities unless otherwise explicitly mentioned. The simulations are performed up to $t^{*}=73$ (in DNS) and $t^{*}=75$ (in LES) with data stored approximately every $t^{*}=1.3$.

\section{Results and Discussion}

Figure 1 shows instantaneous snapshots of the side view of the liquid jet from DNS and LES. In the DNS (Figure 1a), the interface is sharp and is indicated by the zero level iso-contour of the level set function $\phi$. The droplets atomized from the liquid core of the jet and the instability waves formed on the liquid jet column can be clearly seen in Figure 1a. However, 
in the case of LES (Figure 1b), due to the diffused nature of the interface, the iso-contour of $\bar{F}=0.5$ is an indication of where the mean interface could be but not the exact location of the interface. The droplet breakup occurs at large as well as small scales however those at large scales are not visible in the visualization (c.f. Figure 1b) since the interface is diffused. Moreover, a part of the small scale breakup occurs in sub-grid scales and hence such sub-grid droplets are also not visible in the visualisations.

The DNS and LES results revealed two main simultaneous atomization mechanisms: column/bag and ligament/surface breakup. The instabilities that are formed predominantly on the windward side of the liquid jet column generate roll-ups that continue to grow along the jet finally forming bag-like structure. Such structures break, forming varying sized droplets, thus, called column/bag breakup [9]. In addition, ligaments are seen stripping off the sides of the liquid column in the simulations. This phenomenon is seen as surface rupture in the literature [9], thus, its name ligament/surface breakup. Such ligaments undergo further breakup into droplets due to Rayleigh-Plateau instability. These observations of the breakup mechanisms are consistent with the literature [9, 11].

The instability waves generated on the windward side of the liquid jet column are responsible for the droplet and ligament breakup. The plots of the contour of liquid volume fraction from DNS and LES shown in Figure 2 clearly depicts these instability waves as the corrugations on the windward side of the liquid jet. These contour plots are obtained at the mid-plane location along the spanwise direction (direction perpendicular to both liquid injection and crossflow) in the computational domain. In fact, the waves are 


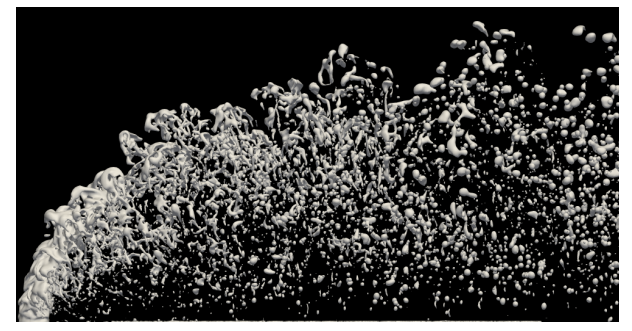

(a) DNS

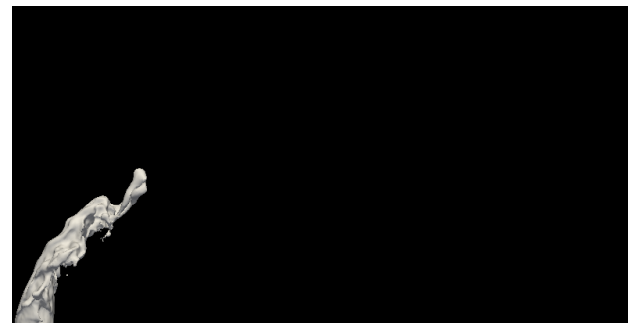

(b) LES

Figure 1: Instantaneous snapshots of the side view of the atomizing liquid jet obtained from DNS and LES.

profoundly visible in the DNS result (Figure 2a) due to the sharp interface capture while the diffused interface capture is demonstrated in the LES result (c.f. Figure 2b). The reader is referred to the study of Asuri Mukundan et al. [18] for a detailed analysis of these instability waves.

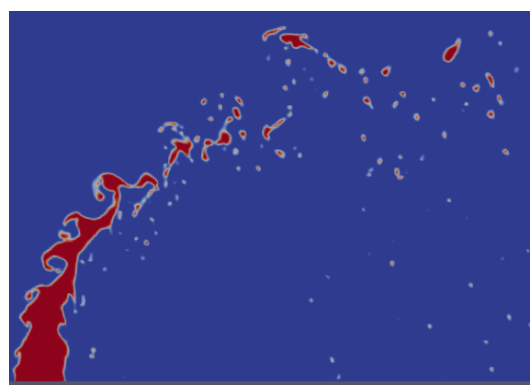

(a) DNS

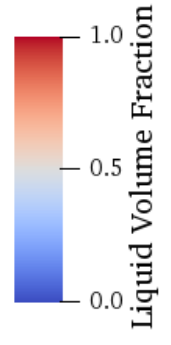

1.0 .0 苞

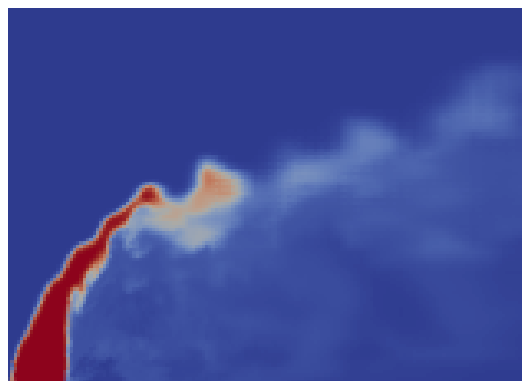

(b) LES

Figure 2: Instantaneous snapshots of liquid volume fraction from DNS and LES in the near-injector region.

Next, we analyze the mean jet penetration obtained from the DNS and LES results. The mean jet penetration and bending gives a detailed representation of the jet penetration probability which determines the size of the combustion chamber in aero engines. In the DNS, the mean jet penetration 
is obtained from the average of several instantaneous snapshots of the visualization of the side view of the liquid jet. The rationale behind such an averaging procedure is to be consistent with that employed for the shadowgraphy images from experiments. In the LES, since the interface is diffused, the precise location of the interface is not clearly determined. Thus, the upper jet plume boundary is determined as the locus of points for each line in the jet injection direction where the liquid volume fraction decreases below a threshold $\delta \%$ of the local maximum value. In this paper, $\delta=5$ is chosen to determine the jet upper boundary. This method has been suggested for experimental analysis of the trajectory [3] and has been implemented numerically [33, 34]. Figure 3 shows the mean jet penetration from DNS and LES compared with the experimental correlations for validation. The jet plume boundary obtained from simulations are compared with the two experimental correlations: Wu et al. [5] (valid in near-injector regions) given as $z / D_{j}=1.37\left(q x / D_{j}\right)^{1 / 2}$ and from Stenzler et al. [6] (valid in far-injector regions) given as $z / D_{j}=2.63 q^{0.442}\left(x / D_{j}\right)^{0.39} \mathrm{We}_{\mathrm{c}}^{-0.088}\left(\mu_{j}^{\exp } / \mu_{\mathrm{H}_{2} \mathrm{O}}\right)^{-0.027}$ where $\mu_{j}^{\text {exp }}$ correspond to the dynamic viscosity of the liquid used in the experiments. On analyzing Figure 3, we see that simulation results qualitatively agree with the experimental correlation of Stenzler et al. [6] (gray dashed line) that that of Wu et al. 5] (green solid line). Such an agreement is consistent with the observation from the literature [9]. As remarked by Herrmann et al. [11, the low value of the liquid-to-gas density ratio of 10 used in this work (compared to the density ratio of 816 in experiments) is prone to under-prediction of the jet penetration and bending in the simulations for the near-injector correlation of Wu et al. [5]. 


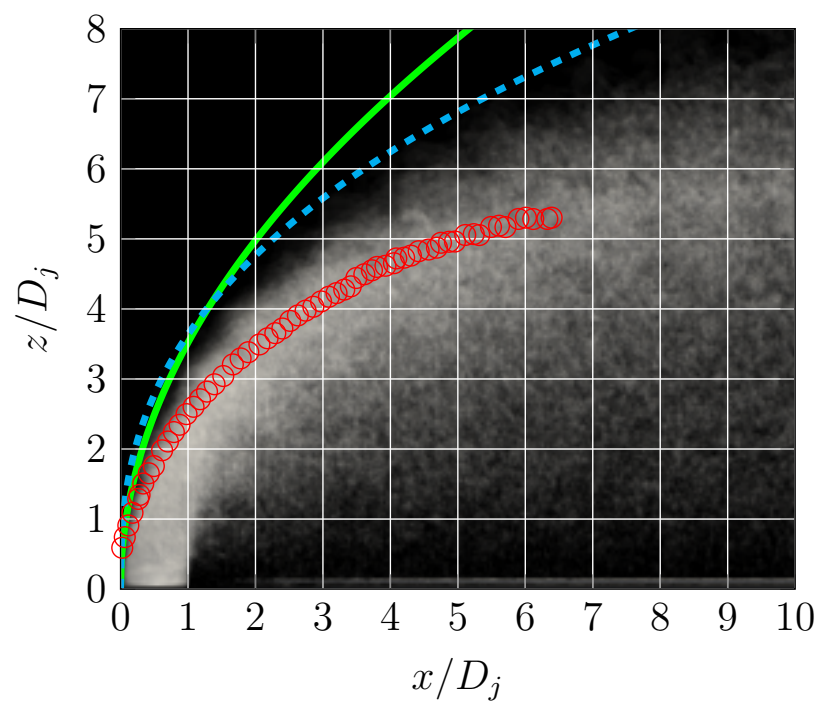

Figure 3: Averaged side view snapshot of the liquid jet in crossflow from DNS with experimental curve fit. DNS(averaged image), LES (b) Wu et al. [5] $\square$, and Stenzler et al. [6] $-=-$.

Besides the jet penetration and bending, the DSD is a vital quantity to ascertain the quality of the primary atomization. Moreover, it is useful for primary atomization modeling in which droplets are injected along a specific liquid core path with a given velocity [35]. The DSDs are obtained from the DNS and LES by post-processing the data after the total mass in the computational domain is stabilized to indicate statistical steady state. The droplets/liquid structures from the DNS are first identified using a connected component labelling (CCL) liquid structure detection algorithm in which the cells belonging to each single liquid structure (i.e., droplets) are tagged and labelled forming a cluster of cells for each liquid structure. The droplets and their attributes are then collected for each structure (from all its comprising cells). The attributes include volume, surface area, volume averaged 
coordinates of droplet centroid, and volume averaged velocity components at centroid. An equivalent spherical diameter of each droplet is obtained from its volume. The droplets are then sampled at specific downstream locations $x / D_{j}$. The DSD is then generated by binning the drop diameters into 20 bins of equal size in terms of $\log \left(d_{\text {drop }} / D_{j}\right)$ where $d_{\text {drop }}$ represents the drop diameter. A total of 10,282 droplets were collected over all sampled time steps from the DNS. In the case of LES, due to the diffused nature of the interface, no grid reslved droplets (drops larger than mesh spacing) are captured. However, the sub-grid droplets are captured whose diameter values are determined from the Equation (7). The DSD is then generated by these diameter values using the same procedure as that of the DNS.

The droplets are sampled at the locations $x / D_{j}=5,10,15,20,25,30$ from the DNS and LES results. Figure 4 shows the individual plots of the DSD from DNS and LES sampled at $x / D_{j}=20$ along with their log-normal distribution fits (solid lines in the plots). It can be seen that the DSD span over a finite range of the droplet diameters that are within the limits of DNS and LES mesh spacings. A log-normal fit (solid lines in Figures $4 \mathrm{a}$ and $4 \mathrm{~b}$ ) to the DSD from the DNS and LES is observed to be a good model as also observed by Herrmann [9]. It is to be remarked that the fitting is made based on the respective simulation data range, hence, the fitting law parameters are different for DNS and LES. The drops with diameters $d_{\text {drop }}<2 \Delta x$ are not well resolved in the DNS while the drops larger than $\Lambda_{20}$ (mesh spacing at the sampling location $x / D_{j}=20$ ) are not found in the LES. Since the DNS captures the mesh resolved droplets and LES characterizes the sub grid droplets by construction of each simulation framework, the combined DSD 
would determine the physical DSD. The rationale behind this idea is that either approach (i.e., DNS and LES) is good for one range of drop sizes so a combined DSD will give a good estimate of the true measure of the range of the droplet diameters belonging to resolved as well as sub-grid scales. This resulted in a direct comparison of the DSD between DNS and LES as shown in Figure 4c. This figure revealed that there is an overlap region of finite size $\left[0.08 D_{j}, 0.2 D_{j}\right]$ for the droplet diameters. To get the model for the physical DSD, a log-normal distribution is found to be a reasonable fit as shown by the solid line in Figure 4c capturing the large well resolved droplets from the DNS and small sub-grid droplets from the LES.
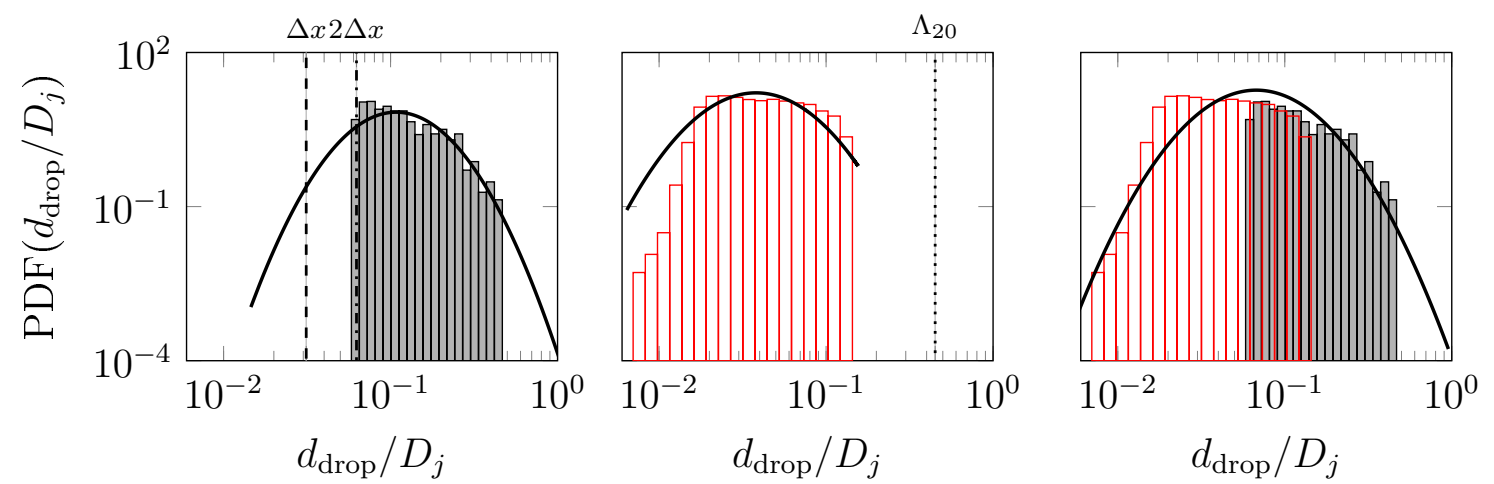

(a) DNS drop size distribu-(b) LES drop size distribu- (c) Combined drop size distion tion tribution

Figure 4: Drop size distribution from DNS

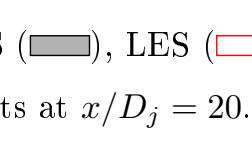

Now, we analyze the plots of the DSDs from the DNS and LES sampled at the other downstream locations shown in Figure 5. At all sampling locations, there exists a finite sized overlap region in the DSD: for $x / D_{j}=5$ it is 
$\left[0.055 D_{j}, 0.167 D_{j}\right]$ (Figure $5 \mathrm{a}$ ), for $x / D_{j}=10$ it is $\left[0.0563 D_{j}, 0.166 D_{j}\right]$ (Figure $5 \mathrm{~b}$, for $x / D j=15$ it is $\left[0.0579 D_{j}, 0.149 D_{j}\right]$ (Figure $5 \mathrm{c}$ ), for $x / D_{j}=25 \mathrm{it}$ is $\left[0.0584 D_{j}, 0.127 D_{j}\right]$ (Figure $5 \mathrm{~d}$ ), and for $x / D_{j}=30$ it is $\left[0.0476 D_{j}, 0.131 D_{j}\right]$ (Figure 5e). Overall, it can be seen that the length of the overlap region reduces downstream until the last sampling plane of $x / D_{j}=30$ which could indicate generation of a bimodal distribution of large and small droplets.

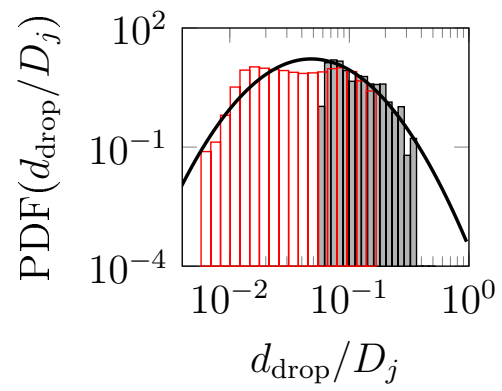

(a) $x / D_{j}=5$

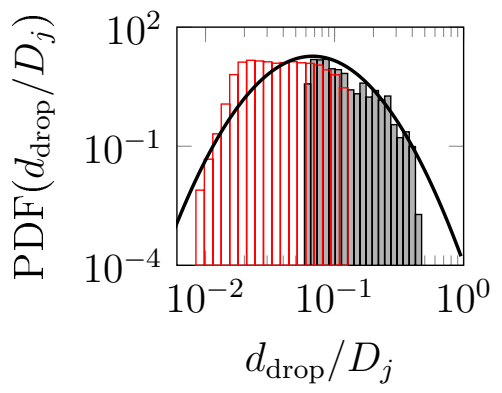

(d) $x / D_{j}=25$

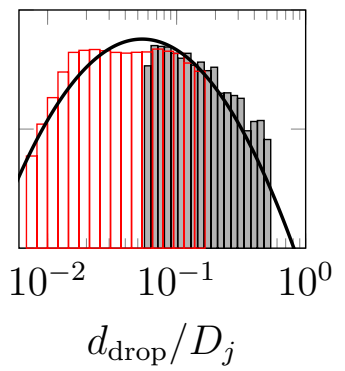

(b) $x / D_{j}=10$ (c) $x / D_{j}=15$

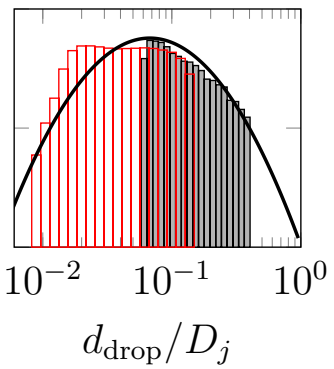

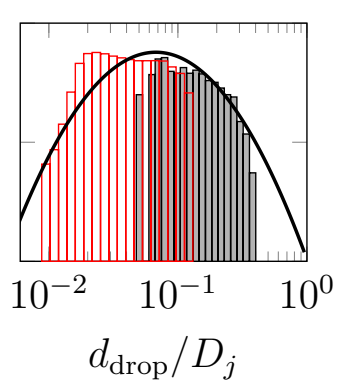

(e) $x / D_{j}=30$

Figure 5: Drop size distribution from DNS $\square$ and LES $\square$ at different downstream sampling locations along with their respective log-normal fits $\square$.

On one hand, the detection of drops with $d_{\text {drop }}>0.1 D_{j}$ shows the presence and importance of large drops that are not captured by the LES. On the other hand, the presence of sub-grid droplets shows the significant pres- 
ence of small drops not captured by DNS. This wide range of scales needs to be considered when modeling primary atomization for liquid jet in crossflow configurations. The agreement between the DNS and LES in the overlapping (or shared) range at all sampling locations (c.f. Figure 5) suggests that the sub-grid distribution observed from the LES can be reliable. The scales investigated by DNS and LES are shown in Figure 6 displaying the schematic representation of the ideal limits of the droplet sizes depicted by the hatched region. This highlights the advantage of a combined DNS and LES study allowing to extract a wide of range of drop sizes for analyses and modeling of primary atomization processes.

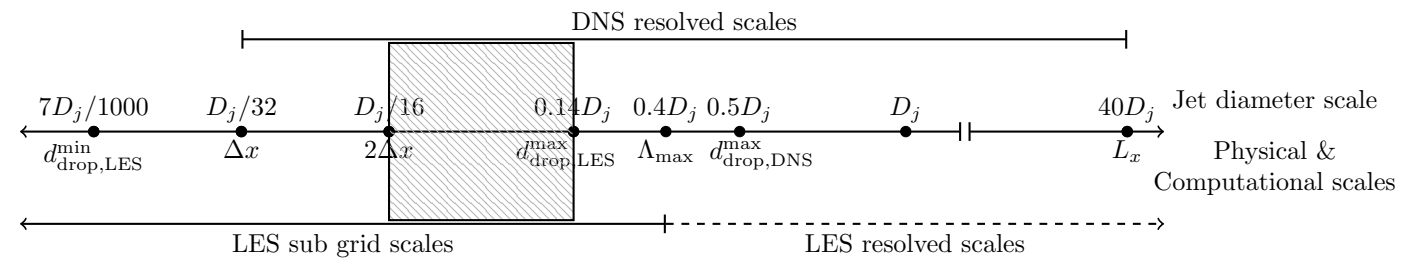

Figure 6: Schematic of range of length scales of motion resolved by DNS and LES at $x / D_{j}=20$ with shared length scales shown in hatched region.

\section{Conclusions}

Results from direct numerical simulations (DNS) and large eddy simulations (LES) of the primary atomization of turbulent liquid jet injected into subsonic gaseous crossflow environment have been presented. The liquid/gas interface has been reconstructed using a sharp coupled level set volume of fluid (CLSVOF) method in DNS while a diffused volume of fluid (VOF) method has been used in LES. The incompressible Navier-Stokes 
equations have been solved directly without a sub-grid model in the DNS and a $\Sigma$ - $Y$-PDF approach with WALE sub-grid scale model in the LES. Two simultaneous atomization mechanisms have been observed in the simulations: column/bag breakup and ligament stripping/surface breakup at the sides of the jet. The column instability growth has not been captured in the LES due to diffused nature of the interface. The liquid jet penetration and bending obtained from the DNS and LES qualitatively agree with the experimental correlation however under-predicting the bending. This under-prediction is attributed to the lower liquid-to-gas density ratio operating condition used in the simulations. Despite not being able to capture the interface dynamics exactly, the LES-PDF breakup predicts a similar sub-grid DSD as the DNS in the range $2 \Delta x<d_{\text {drop }}<\Lambda$. The DSDs obtained from DNS and LES follow log-normal distributions based on the respective simulation data. A combination of the DSDs from DNS and LES offers a wide range of length scales and gives a log-normal distribution as a good fit. The results from this work are intended to be used as a starting point for further investigations of the effect of density and viscosity ratio on the atomization characteristics at higher mesh resolutions.

\section{Acknowledgments}

The funding for this project from the European Union's Horizon 2020 research and innovation programme under the Marie Skłodowska-Curie grant agreement $\mathrm{N}^{\circ} 675676$ is gratefully acknowledged. The computing time at CRIANN (Centre Régional Informatique et d'Applications Numériques de Normandie) under the scientific project No. 2003008 and at GENCI-[TGCC/ 
CINES/IDRIS] (Grant2019-2613) are also gratefully acknowledged. The authors also acknowledge funding by the UK's Engineering and Physical Science Research Council support through the grant EP/S001824/1 (KV, GT).

\section{References}

[1] C. Aalburg, B. van Leer, G. M. Faeth, K. A. Sallam, Properties of nonturbulent round liquid jets in uniform gaseous cross flows, Atomization and Sprays 15 (2005) 271-294.

[2] C.-L. Ng, R. Sankarakrishnan, K. A. Sallam, Bag breakup of nonturbulent liquid jets in crossflow, International Journal of Multiphase Flow 34 (2008) 241-259.

[3] C. T. Brown, V. G. McDonell, in: Proceedings of the ILASS Americas, 19th Annual Conference on Liquid Atomization and Spray Systems, May 23-26, 2006, Toronto, Canada.

[4] R. K. Madabhushi, M. A. M. Y. Leong, C. T. Brown, V. G. McDonell, in: Proceedings of the ILASS Americas, 19th Annual Conference on Liquid Atomization and Spray Systems, May 24-26, 2006, Toronto, Canada.

[5] P. K. Wu, K. A. Kirkendall, R. O. Fuler, A. S. Nejad, Breakup processes of liquid jets in subsonic crossflows, Journal of Propulsion and Power 13 (1997) 64-73.

[6] J. N. Stenzler, J. G. Lee, D. A. Santavicca, W. Lee, Penetration of liquid jets in a cross-flow, Atomization and Sprays 16 (2006) 887-906. 
[7] S. B. Leask, V. G. McDonell, S. Samuelsen, Critical evaluation of momentum flux ratio relative to a liquid jet in crossflow, Atomization and Sprays 28 (2018) 599-620.

[8] M. Broumand, M. Birouk, Liquid jet in a subsonic gaseous crossflow: Recent progress and remaining challenges, Progress in Energy and Combustion Science 57 (2016) 1-29.

[9] M. Herrmann, Detailed numerical simulations of the primary atomization of a turbulent liquid jet in crossflow, Journal of Engineering for Gas Turbines and Power 132 (2010) 061506-1-061506-10.

[10] M. Herrmann, The influence of density ratio on the primary atomization of a turbulent liquid jet in crossflow, Proceedings of the Combustion Institute 33 (2011) 2079-2088.

[11] M. Herrmann, M. Arienti, M. C. Soteriou, The impact of density ratio on the liquid core dynamics of a turbulent liquid jet injected into a crossflow, Journal of Engineering for Gas Turbines and Power 133 (2011) 061501.

[12] S. Ghods, M. Herrmann, in: Proceedings of the ILASS Americas, 25th Annual Conference on Liquid Atomization and Spray Systems, May 3-5, 2013, Pittsburgh, PA, USA.

[13] M. Owkes, M. G. Pai, O. Desjardins, in: Proceedings of the AIAA SciTech Forum, January 13-17, 2014, National Harbor, MD, USA.

[14] Y. Gopala, Breakup characteristics of a liquid jet in subsonic crossflow, Ph.D. thesis, Georgia Institute of Technology, 2012. 
[15] X. Li, M. C. Soteriou, High fidelity simulation and analysis of liquid jet atomization in a gaseous crossflow at intermediate weber numbers, Physics of Fluids 28 (2016) 082101-1-082101-35.

[16] X. Li, M. C. Soteriou, Detailed numerical simulation of liquid jet atomization in crossflow of increasing density, International Journal of Multiphase Flow 104 (2018) 214-232.

[17] A. Asuri Mukundan, T. Ménard, A. Berlemont, J. C. Brändle de Motta, M. Herrmann, in: Proceedings of the ILASS Americas, 30th Annual Conference on Liquid Atomization and Spray Systems, May 12-15, 2019, Phoenix, AZ, USA.

[18] A. Asuri Mukundan, T. Ménard, M. Herrmann, J. C. Brändle de Motta, A. Berlemont, in: Proceedings of the AIAA SciTech Forum, January 610, 2020, Orlando, FL, USA.

[19] T. Ménard, S. Tanguy, A. Berlemont, Coupling level set/VOF/ghost fluid methods: Validation and application to 3D simulation of the primary break-up of a liquid jet, International Journal of Multiphase Flow 33 (2007) 510-524.

[20] S. Osher, J. A. Sethian, Fronts propagating with curvature-dependent speed: Algorithms based on Hamilton-Jacobi formulations, Journal of Computational Physics 79 (1988) 12-49.

[21] G. D. Weymouth, D. K.-P. Yue, Conservative Volume-of-Fluid method for free-surface simulations on Cartesian-grids, Journal of Computational Physics 229 (2010) 2853-2865. 
[22] G. Vaudor, T. Ménard, W. Aniszewski, M. Doring, A. Berlemont, A consistent mass and momentum flux computation method for two phase flows. Application to atomization process, Computers \& Fluids 152 (2017) 204-216.

[23] A. Asuri Mukundan, T. Ménard, J. C. Brändle de Motta, A. Berlemont, A 3D Moment of Fluid method for simulating complex turbulent multiphase flows, Computers \& Fluids 198 (2020).

[24] S. Navarro-Martinez, Large eddy simulation of spray atomization with a probability density function method, International Journal of Multiphase Flow 63 (2014) 11-22.

[25] L. Valiño, A field monte carlo formulation for calculating the probability density function of a single scalar in a turbulent flow, Flow, turbulence and combustion 60 (1998) 157-172.

[26] R. Lebas, T. Ménard, P. A. Beau, A. Berlemont, F. X. Demoulin, Numerical simulation of primary break-up and atomization: Dns and modelling study, International Journal of Multiphase Flow 35 (2009) $247-260$.

[27] B. Duret, J. Réveillon, T. Ménard, F. X. Demoulin, Improving primary atomization modeling through DNS of two-phase flows, International Journal of Multiphase Flow 55 (2013) 130-137.

[28] H. Jasak, A. Jemcov, Z. Tukovic, in: International workshop on coupled methods in numerical dynamics, volume 1000, IUC Dubrovnik, Croatia, pp. 1-20. 
[29] F. Nicoud, F. Ducros, Subgrid-scale stress modelling based on the square of the velocity gradient tensor, Flow, turbulence and Combustion 62 (1999) 183-200.

[30] H. G. Weller, A new approach to VOF-based interface capturing methods for incompressible and compressible flow, Technical Report TR/HGW/04, OpenCFD Ltd., 2008.

[31] P. E. Kloeden, E. Platen, Numerical solution of stochastic differential equations, volume 23, Springer Science \& Business Media, 2013.

[32] C. W. Gardiner, Handbook of stochastic methods, volume 3, Springer Berlin, 1985.

[33] A. Andreini, C. Bianchini, S. Puggelli, F. Demoulin, Development of a turbulent liquid flux model for Eulerian-Eulerian multiphase flow simulations, International Journal of Multiphase Flow 81 (2016) 88-103.

[34] S. Puggelli, L. Mazzei, A. Andreini, in: Proceedings of the 1st Global Power and Propulsion Forum GPPF, January 16-18, 2017, Zürich, Switzerland, pp. 1-9.

[35] M. Behzad, A. Mashayek, N. Ashgriz, in: Proceedings of the ILASS Americas, 22nd Annual Conference on Liquid Atomization and Spray Systems, May 16-19, Cincinnati, OH, USA, ILASS2010-112. 